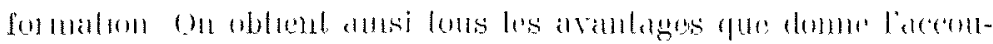

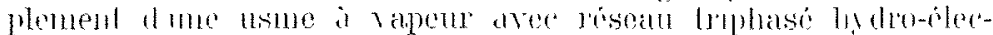

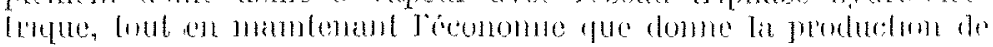

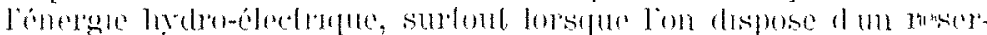
ron ligelaniuleque.

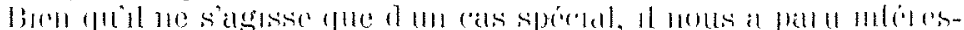

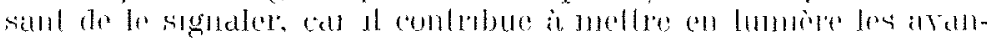

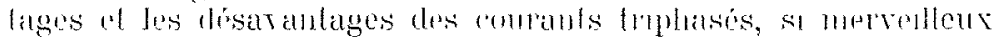
lans loules lours applicaloms.

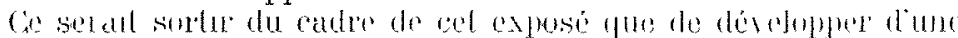

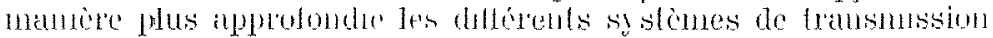

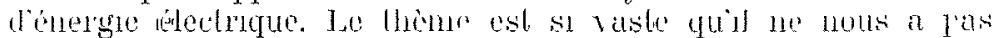

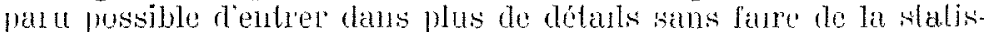

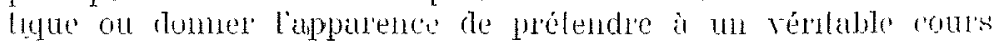

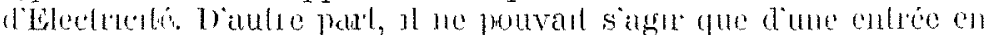

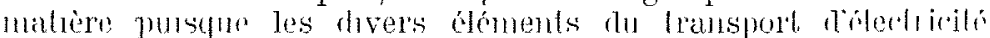

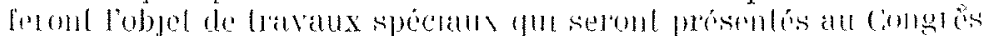

\section{USINE HYDRO-ÉLECTRIQUE DE SCOTLAND}

La Lincas l'ower $C^{\prime \prime}$ vient le terminer l installation d'uno usine hydro-électrique a scotland (Conneclicut), sul la shetuclict River. La hauteur normale de la chute ast de imfig, et est obtenue au moyen d'un barrage établs atu travel's de la rivière, dans le prolongement do l'usine $\left(^{*}\right)$.

Sur la rive gauche, le barrage forme déversoir sur 100 pieds $\left(^{*}\right)$, dont les 211 premier's, à la suite de l'usme, sont on béton massil, tandis que los 80 suivants sont (n jueton armé creux, du type de la Ambursen-IIydraulic Constuction $\mathrm{C}^{\circ}$, donl la figure 1 ci-jointe replésento une section transversalc. Les contreforls intéricurs ont unc épaisscur fui varie de 18 pouces a la base à 12 pouces au sommet, leur dislance d'axe en axe est de 10 pieds. L'épaisseur du parement aval est de 20 pouces; celle du parement anont varic de 20 pouces a la base a is pouces au sommet. Ces deux paroments sonl en béton dose a laison de parties de ciment pour 23 de sable. 'ar dessus, ust un enduil, de 1 pouce d'épaisseur, dosé à 1/2. Des lrous, ménacrés dans; la dalle de fondation, empêchent toute sous-pression.

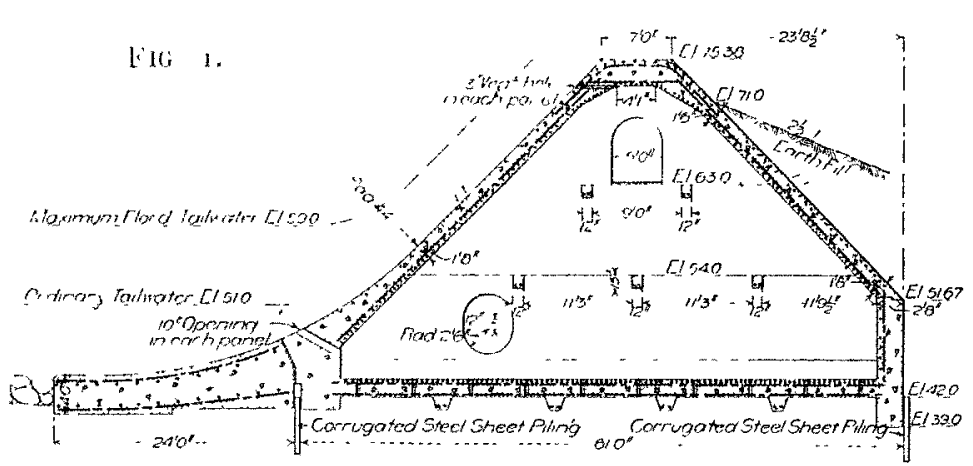

Liemploi de deux lypes différonts pour le déversoir pro. vent de ce que, daus le projet primilif, on complait ne fairo quiun doversoir massil, mais, au cours des travalux, on sappercut que le sol de fondalion qui, a l'emplacement de l'usine, etait constitué par du locher solide, so contmuail 'nsulte par un terain glaveleux qui parut insul'lisant pour nu massif plein en béton, el l'on résolut de modılier le typo primitif, deja en parli conslrut, of contmuer avec un barrage creux.

Des hausses moljiles, installecs sur la crete du devel'soin, permettent de relever de It pultecs le plan deau amont.

sur la live droite, le larrage est constilue par une diguo "n terre, arrasée a la colo ?0, arec ime centrale en béton anmé, do 170 piods de lonsueur. Les matériaux gui consti.

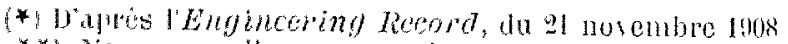

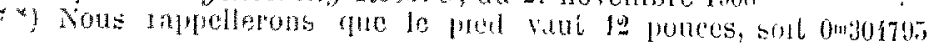

tuenil cetto digue proviement dos fondations de la palto centrale du barrage. Les parcments sont inclinés à $1 / 2$. largeur it la crêle esl do 18 picds.

Dans la partic contrale, le barrage est represonté jar figure 2, et ost coustituó par 5 vannes 'Taintel', de 90 pleds d'ouvorture, qui s'appuient sur des piliers de a pieds d'épaic seul. L'eau qui passo sous les vamnes, lorsque celles.ci sont levés, tombo dans une chambre d'amortissemen

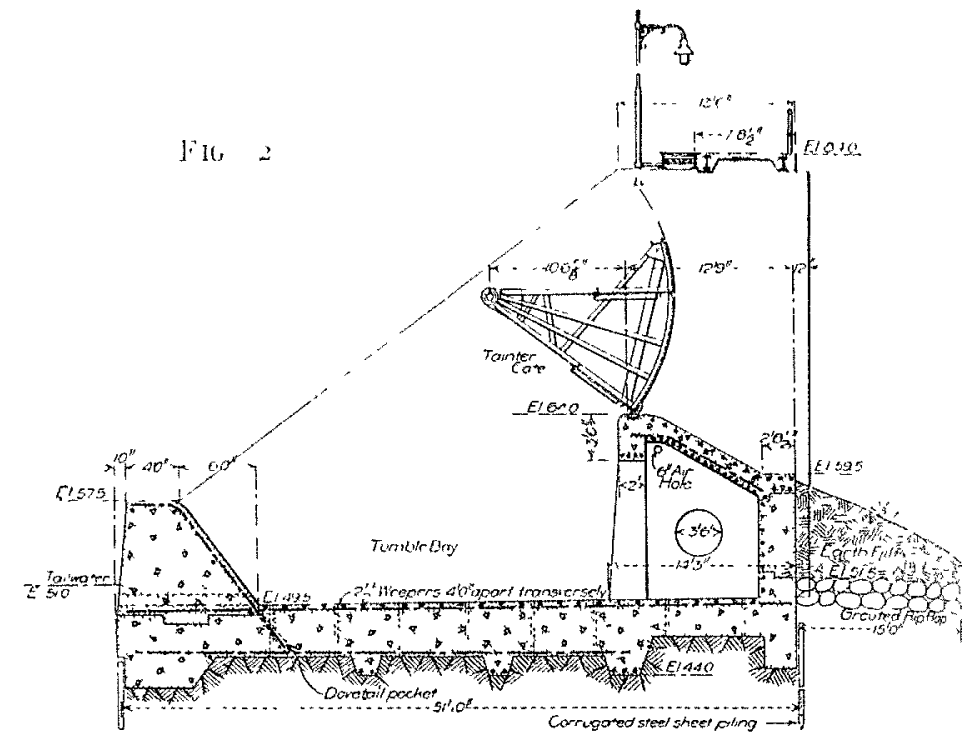

(/umble $b(t y)$. Ce dispositil est tout à fait analogue a tem ifui a cié employ é pour les usmes de Grand hapjols, But

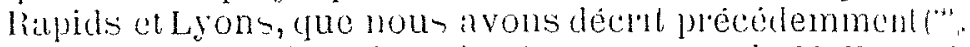

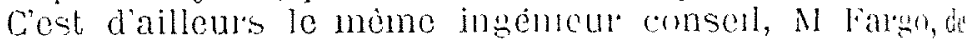
Jaclison, qui les a étudies.

Chacuo vanne Tainter a 13 prodis de hauleur, of os rein.

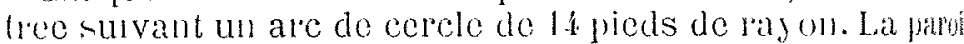
en contact aves l'oau est en listo d'acier, do //1 de poute depalsseur. Les vamnes sont levers an mojen lo dew chames, allachóes à 10 pied- des extrimitos, ol mues, solt

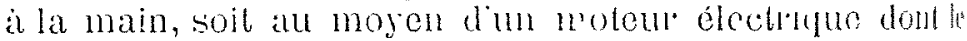
courant provient de l'exclatrice de lusine l'élanchelí est obtenue a la partic inléricure par interposition do mindriers en bois, fixes au bas do la vanne et au sonmel du deverson en bélon, el sul les cótus par des bandes de cautchouc. Afin de dimmuer l'usure de colles-ci. on al dsposć, sur les parnis dos piliers, dos ares do cercle mflalliques bien dressós, contro lesquellos cllos viennent frotter.

La chambre d'amoltissenenl se conthnue pal un arréete radler, de 30 pieds de longlleur, aupuel fail sute, nip 70 autres pieds, un dallage spécial contre les alfoulle ments. Colui-ci est compose de dalles on bóton amé, te 16 < $17 \%$ pleds, ot do 27 polces à 21 pouces d'épaisseur assemblées les unes aux autres do la manière suivante: Deux tiges mélalliques, recourjées en $U$, l'une verlicale ment, lautre holizontalement, sont scellees dans chatu dalle à chaque jontl. De plus, l'une des paroos, à chaqu joint, a une contrbure concave, landis que la paroi opposen est convexe, de surte que la dalle a une certaino rossen. blance avee un livle, d'ou le nom de book-slab quon luia donnci

Des l'adeaux, solidement altachés sur chacuc rive at thi estacade permeltant leur muuvemont vortical, dirigent les (i)lps flotlants sur le déversoir.

Lin amoul de la digue de terr', aussi qu'en amontef ean aval du ballage en bolon, on a hatlu une rangée de pat planches inctalliques poul ampechol tout glissement ill sous-sol.

lendant les travaux, les diverses parlies du barrage ond ćtó entourées de batal'deaux composés do deux langées do palplanches en chalaignier, rle 2 pouces d'épaisseur, battues

(**) Vure La Houble Blanche de mand 1 tod. 
\& $8 \%$ pieds d'écartement. I'espace intermédiaire a ète unsute bourré de gravier.

Le batiment principal de l'usine génératrice à 71 pieds de longueur sur 29 de largeur, il ne contient que le matériel électrique, toute la partie hydraulique so trouvant a l'extélıur, comme lo montre la figure 3.

I, usine comprond 3 groupes électrogènes princupaux, composés chacun d'une turbine double, directement accouplée à un alternateur de $400 \mathrm{~km}$, sous 3000 volts, el d'une axcilatrice de $40 \mathrm{kw}$. Trois transtormateurs de $400 \mathrm{kw}$ ele ment la tension du courant à 23000 volts. Celui-ci est ainsi curojo à Nolwich, distant de 17,6 kilométres.

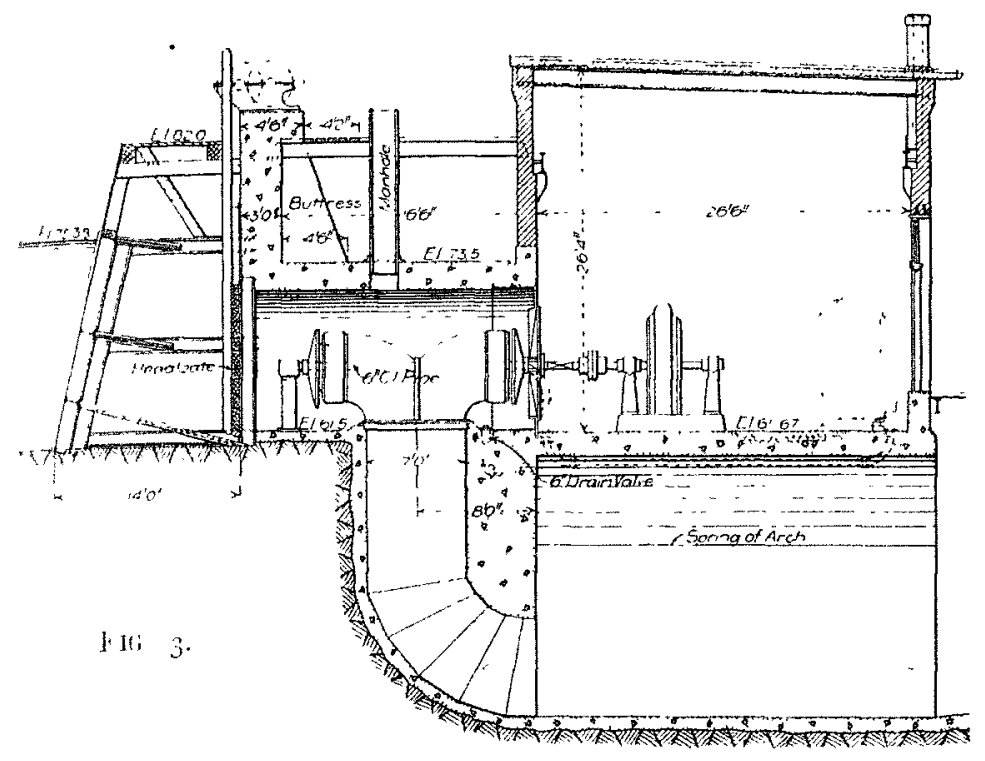

Remarque. - Les vamnes 'Tainlel', assez cmploj ćes en Amérique, 11e sont qu'une variante du type général de barrage mobile a segment dont on trouve plusieurs aulres cxemples en Autrichc et en Illemagne. En France, ce s.yslème cst encore peu répandu, bien qu il semble que ce solt dans molre pays qua l'idée première y ant pris naissance kn effel, dans les Innales des Ponts-et Chaussees de 1908 (juillol-aoùl), M. Gurbal, ingénieur en chef des Ponts-elChaussées, en a revendiqué la paternité, el a dommé la desrription des portes écuilibrées des ecluses du lez, sul lo tálal du Rhòne à Celte, qui ont ólé construites par lui clo 1886 à 1887.

Chaque porte consiste essentiellement en une vanne, construite sous la lonme d'un segment cylindrique tournant autour d'un axe horizontal, on tout point scmblable a la vanne Tainter de la figure 2 . Ello n'en diffère qu'en ce qu'elle était équilibrée à peu près poul' toules ses positions, au moyen d'un dispositif particulier. Depuis plus de 20 ans qu'elle est en service, celte vanne n'a cessé de fonctionner avec la plus stande régularité, el presque sans aucun entretion.

Dans les vannes à sergtnent.l'axe du segment cylundrique coincide généralement avec laxe de l'otation; il en résulte que la poussée de leau passe yar l'axe de rotation. Il y aurail cependant avantage à laire passer l'axe du segmenil tur peu en dessus de l'axe de rotation; de la sorte, lo moinont de la poussée de l'eau par lapport à cel axo ne selat fas nul, el aurail poul effel de dminuer leffort nécessaire i la levée de la vanne, surtoul au départ oi le cocfficiant de frottement est le plus élevé, et l'effort nécessaire le plum considérabli.

II. B.

Nous rappelons que tout ce qui concerne la Rédaction doit être adressé au rédacteur en chef, $M$. COTE, 24, rue Sully, a LYON, et que tout ce qui concerne l'Administration doit être adressé aux éditeurs, MM. GRATIER et REY, 23, Grande Rue, à GRENOBLE.

\section{APPAREIL POUR LA MESURE DU GLISSEMENT DES MOTEURS ASYNCHRONES (*)}

Lad vilesse des moluurs qu'almentent lus réseaux de distribuLion électrique ì couranls alternatifs dépend de la trequence $f$ du du courant employé. Quand un moteur lourne à vide, sa vileste est sensiblement celle du synchromsme, c'est-it-dire que la duree dun tour est égale a $\frac{n}{f}$ seconte, "dant le nombre de parres de pôles de ce moleur.

Pour les moteurs la bye le flus courant, lavitesse du synchronrsme ne se trouve a peu plés rodisée que dans la marche it vide. Lorsque la charge augmente, la vilesse dimmue: un lit que le moteur glisse.

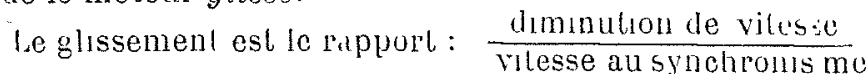

Par exemple, quand un moleur a leax pòles, alımenté à la fréquence 50 , ne lourne plus qu'ì 2700 tours par inmule, son glissement esl égal à $\frac{3.000-2.700}{3.000}$, c'esl-à-chre igal à it pour 100

ln peul encore explimer lo glissement en foncuun de latugmenlation de la duréc d'un tour. Si nous désignous celle augmenlation par z, le glissement sera très sensiblement cgal au lapport:

lurée dun tour au synchronismo

Deux mélhodes bien connues permettent de mesuref le glissemenl.

La plus smple comsiste à intercaler un amporemetre dins he circuil du rotor, el a chronoméller les oscillalmas de l'aigulll. Mans elle n'est pas applicable à une lrès mportanle calégorne do moteurs: les moteurs en court crreulf de llum, la mesure esl

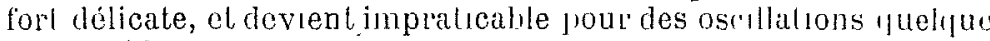
peu rapides.

Ivec la scconde méthode, dile aslrobuscoplypen, on place, il lane des cxtrèmitós de larbro du moleur, un disque encarton sur lequal sont peinls des secteurs noirs sur fond blanc, en nombre égal à celur des poles du moleul: Le disque bol celand par une lampe qu'alimente le l'éseau, 1 semble Lourner on sens luverse du moleur, el la vilusse angulare de celle rotation apjurente correspond au glissement.

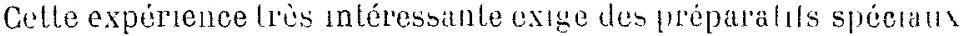
qui rendent son exćculion difficale. Enfin, comme pour le premier procédé, dès que le glissement saccentut, louk mesure devent. impossible.

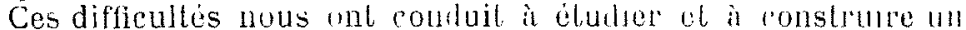

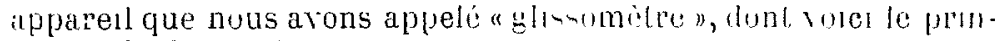
cipe el la description sommatre :

Principe de l'Appareil. - Supposmus lixe, ch buat darble l'un moleur, un cylndre cu curre purtaul sur ba pémphérne les sectours isolanis $a$, $a, a, a$, commo lindiqu" la figure 1 In frotleur $b_{4}$ repose on permanchce sur le curre, un anles, $b_{2}$, prend contact, lantól sur le curver, el lanbot sur lisolant.

lie rlsposilil oure el ferno allemalivement le rircunt rine

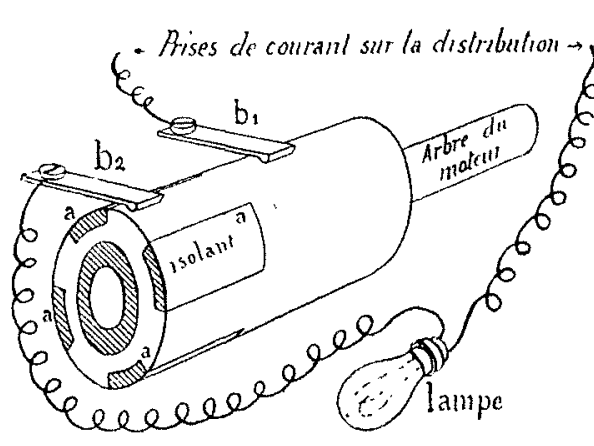
lampe a lucandescence. almontée par le courant 6 du léscau sir lefuel th lroule branchedranoleur de nombrr des seeleurn rolants rel jors ipal all nombre de polew la mo teur; 1! s'ansl lone, daus 1. ras le la figure, lium moleur à qualre piles. Nous supflusorolls ell 1)ubr $f 0$.

$\Delta u$ sinchionsome, lat IiI) durée flun lour lo moteur - - $1 / 100$ de seronde, Il sicoule donc, entre deux inleriphlions de rourant, 1/100 de sceonde. Dans ces conditums, si le passage d'un secteul cuive sous le frotleul 11 , concide avec un eclal de la lampe, commi:

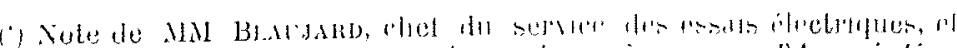

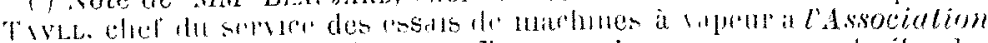

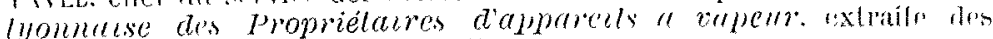
comptes linulum de letio Assoriation. 